\title{
Applications of Hyperdifferential Operators to Quantum Mechanics*
}

\author{
Marvin Miller \\ Department of Electrical Engineering, Purdue University \\ STANLy STEINBERG \\ Division of Mathematical Sciences, Purdue University
}

Received July 15, 1971

\begin{abstract}
In this paper, various applications of the theory of hyperdifferential operators to quantum mechanics are discussed. A concise summary of the relevant aspects of the theory is presented, and then used to derive a variety of operator identities, expansions, and solutions to differential equations.
\end{abstract}

\section{$\S 1$ Introduction}

The purpose of this paper is to point out various applications of the theory of hyperdifferential operators. In particular we will illustrate how this theory can be used to derive a wide variety of operator identities, expansions, and solutions to differential equations of interest in quantum mechanics.

Hyperdifferential operators are differential operators of infinite order with variable coefficients

$$
A=\sum_{m, n=0}^{\infty} a_{m n} z^{m}\left(\frac{d}{d z}\right)^{n}
$$

The feature of these operators which is of particular relevance in applications is the possibility of defining the symbol of $A$,

$$
\sigma A(z, \xi)=\sum_{m, n=0}^{\infty} a_{m n} z^{m} \xi^{n}
$$

in terms of which we can develop a computational calculus, called the symbolic calculus.

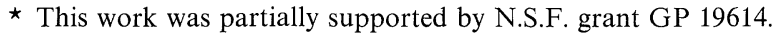


We note that for a hyperdifferential operator $A$ with symbol $\sigma A(z, \xi)$

$$
A f(x)=(2 \pi)^{-\frac{1}{2}} \int_{-\infty}^{\infty} e^{i x \xi} \sigma A(x, i \xi) \hat{f}(\xi) d \xi
$$

where

$$
\hat{f}(\xi)=(2 \pi)^{-\frac{1}{2}} \int e^{-i x \xi} f(x) d x,
$$

is the Fourier transform of $f(x)$. Here we must assume that $f$ has the proper smoothness and growth characteristics. $(x$ is a real variable while $z$ and $\xi$ are usually complex variables.)

In Sections 2 and 3, some results about analytic functionals, hyperdifferential operators, and the symbolic calculus are presented in their most elementary form. In particular, in Section 2 we introduce several representations of analytic functionals (the most important of which is the Fourier-Borel transform). In Section 3, we derive formulas for the symbol of a product of two operators and the transpose of an operator. These formulas are the essential ingredients in the symbolic calculus. A more detailed exposition can be found in $[14,15]$.

In Section 4, the results of Sections 2 and 3 are applied to Weyl's prescription for quantizing a classical function and to the process of normally ordering an operator. Section 5 is devoted to the solution of a partial differential equation which arises in the theory of two photon amplification [8].

To illustrate the basic concepts, we present the following examples. First, if $\hat{f}(\xi)$ is the Fourier transform of $f(x)$ and $g(x, \xi)$ is a polynomial in $x$ and $\xi$, then

$$
g(x, d / d x) f(x)=(2 \pi)^{-\frac{1}{2}} \int e^{i x \xi} g(x, i \xi) \hat{f}(\xi) d \xi
$$

is a hyperdifferential operator with symbol

$$
\sigma g\left(x, \frac{d}{d x}\right)=g(x, \xi)
$$

Next, consider the initial value problem

$$
\frac{\partial f}{\partial t}(x, t)=P\left(\frac{d}{d x}\right) f(x, t), \quad t>0 \quad \text { with } \quad f(x, 0)=f_{0}(x) .
$$

The solution is given by

where

$$
R(t) f_{0}(x)=f(x, t)=(2 \pi)^{-\frac{1}{2}} \int e^{i x \xi} e^{P(i \xi) t} \hat{f}_{0}(\xi) d \xi .
$$

$$
R(t)=e^{t P(d / d x)}
$$


is a hyperdifferential operator with symbol

$$
\sigma R(t)=e^{t P(\xi)} .
$$

In the case of variable coefficients, that is, $P(d / d x) \rightarrow P(x, d / d x)$, this problem is much more difficult, but can be handled using our techniques (see Section 5). In fact, this is one of the main differences between our theory and for instance [3].

The material found in Section 2 is well-known. The material presented in Section 3 is a special case of the material in [15, 16, Section 21]. However, the proofs in $[15,16]$ use the theory of topological tensor products and consequently are far more complicated than our presentation.

The formulas derived in Section 4 appear in the physics literature. What is new is the use of the symbolic calculus to derive them. In particular, the important Formulas (3.4), (3.8), (3.10), and (3.15) which we use extensively in Section 4 appear in the physics literature, $[12,17]$ but are not used in the extensive way we use them. In addition, our derivation of the formulas (4.4), (4.11), (4.14), (4.15), etc. show that these expressions are valid for a wide class of analytic functions. Previously, it was only known that these expressions were valid for the trivial case of polynomials.

Equations like the equation in Section 5 have been discussed extensively $[2,7,8,10,11,13]$. We include this example here to illustrate the general applicability of our technique.

The main point of this paper is to present the precise mathematical theory of hyperdifferential operators and the symbolic calculus in a very elementary form and to then show its general applicability to quantum mechanical problems.

In Section 6 we discuss generalizations of this theory and some of its relationships to other theories.

We remark that the theory of hyperdifferential operators is formally similar to the theory of pseudo-differential operators and Fourier integral operators (see [5, 6]). However, there are many important differences, in particular, pseudo-differential operators are assumed to be of finite order. The functions appearing in the definition of Fourier integral operators, which in our case correspond to the symbol, must satisfy conditions which our symbol usually does not satisfy. These differences come from the fact that pseudo-differential and Fourier integral operators are designed to operate on Sobolov spaces, that is spaces of functions with a finite number of square integrable derivatives, while hyperdifferential operators are designed to operate on spaces of analytic functions.

Although we only discuss the case of one variable $z$, everything easily generalizes to $n$ variables $z=\left(z_{1}, \ldots, z_{n}\right)$, (see $\left.[13,15,16]\right)$. 


\section{§ 2. Analytic Functionals and the Fourier-Borel Transform}

Let $\mathbb{C}$ be the complex numbers and let $E$ be the set of entire functions mapping $\mathbb{C}$ into $\mathbb{C}$. That is

$$
E=\left\{f(z) ; f(z)=\sum \frac{f^{(n)}(0)}{n !} z^{n}, f^{(n)}=\frac{d^{n} f}{d z^{n}}\right\}
$$

where the power series is assumed to converge uniformly on any compact set. Let $E^{\prime}$ be the dual space of $E$, see [14, p. 231], i.e.

$$
E^{\prime}=\{\mu=\mu(z) ; \mu \quad \text { maps } E \text { into } \mathbb{C} \text { and is linear and continuous }\} \text {. }
$$

We write

$$
\langle\mu, f\rangle=\langle\mu(z), f(z)\rangle .
$$

Remark. $E$ and $E^{\prime}$ are obviously linear spaces. If we define

$$
\|f\|_{R}=\sup _{|z|<R}|f(z)|,
$$

then the countable set of norms $\|\cdot\|_{n}$ define a topology on $E$. In fact, $E$ is a complete metric space, see $[14$, p. 57,89$]$. In this topology, $f_{n}(z) \rightarrow f(z)$ if and only if $f_{n}(z)$ converges to $f(z)$ uniformly on compact sets. Also in this topology $\mu$ is a continuous linear functional if and only if

$$
|\langle\mu, f\rangle| \leqq C\|f\|_{N}=C \sup _{|z| \leqq N}|f(z)|
$$

for some $C$ and $N$. Finally there are the usual topologies on $E^{\prime}$ defined by duality.

Remark. We can now consider certain spaces of functions and distributions $\mu$ belonging to $E^{\prime}$. Suppose that $g(z)$ maps $C$ into $C$ (not analytic) and

i) $g(z)=0$ if $|z|>R$ for some $R$,

ii) $\int|g(x+i y)| d x d y<\infty$.

If

$$
\langle\mu, f\rangle=\int g(x+i y) f(x+i y) d x d y
$$

then clearly $\mu \in E^{\prime}$. In fact if $\mu$ is any distribution on the complex plane with compact support, (see [14, p. 255]) then the restriction of $\mu$ to the entire functions belongs to $E^{\prime}$.

At this point we will discuss several representations for elements of $E^{\prime}$ and then prove the necessary details in a later theorem.

Definition.

$$
\hat{\mu}(\xi)=\left\langle\mu(z), e^{z \xi}\right\rangle=\sum_{n=0}^{\infty} \frac{\left\langle\mu, z^{n}\right\rangle \xi^{n}}{n !}=\sum \frac{\mu_{n} \xi^{n}}{n !}
$$


where $\hat{\mu}(\xi)$ is called the Fourier-Borel transform of $\mu$. If

$$
\mu_{n}=\left\langle\mu, z^{n}\right\rangle
$$

then $\mu_{n}$ is called the $n$th moment of $\mu$.

If we set

$$
v=\sum \frac{(-1)^{n} \mu_{n} \delta^{(n)}}{n !},\left\langle\delta^{(n)}, f(z)\right\rangle=(-1)^{n} f^{(n)}(0)
$$

then we see that $v_{n}=\mu_{n}$ and as the polynomials are dense in $E$, we have $\mu=v$.

Remark. If $\mu$ is given by a distribution with support on the real line, then $\hat{\mu}(-i \xi)$ is the Fourier transform of $\mu$.

We now give $\mu$ a representation as a contour integral. In fact, we have

$$
\left\langle\delta^{(n)}, f\right\rangle=(-1)^{n} f^{(n)}(0)=\frac{(-1)^{n} n !}{2 \pi i} \int_{C} f(\lambda) \lambda^{-n-1} d \lambda
$$

where $C$ is any curve enclosing 0 once. Consequently

$$
\langle\mu, f\rangle=\Sigma \frac{\hat{\mu}^{(n)}(0) f^{(n)}(0)}{n !}=\frac{1}{2 \pi i} \int_{C}\left(\Sigma \hat{\mu}^{(n)}(0) \lambda^{-n-1}\right) f(\lambda) d \lambda,
$$

providing the series converges.

Definition.

$$
\tilde{\mu}(\lambda)=\left\langle\mu(z), \frac{1}{\lambda-z}\right\rangle=\Sigma \mu_{n} \lambda^{-n-1}
$$

and is called the Cauchy (or Fantappie) representation of $\mu$.

Definition.

$$
\begin{gathered}
\operatorname{Exp}=\left\{f(z) ; f(z) \text { is entire, }|f(z)| \leqq C e^{N|z|} \text { for some } C \text { and } N\right\} . \\
A=\{f(z) ; f(z) \text { is analytic for }|z|>R \text { for some } R>0, \text { and } \\
\left.\qquad \lim _{z \rightarrow \infty} f(z)=0 .\right\}
\end{gathered}
$$

where if two functions are equal in some neighborhood of $\infty$, then they are to give the same element of $A$.

Remark. Exp and $A$ can be made into topological linear spaces in a standard way (see $[14$, p. 232 , ex. $22.5 ; 15$, p. 19-01]). 
Theorem 1. The following maps are $1-1$ and onto:
i) $\mu(z) \rightarrow \hat{\mu}(\xi)=\left\langle\mu(z), e^{z \xi}\right\rangle=\Sigma \frac{\mu_{n} \xi^{n}}{n !}$,
$E^{\prime} \rightarrow \operatorname{Exp}$,
ii) $\quad g(\xi) \rightarrow \mu=\Sigma \frac{(-1)^{n}}{n !} g^{(n)}(0) \delta^{(n)}$,
$\operatorname{Exp} \rightarrow E^{\prime}$,
iii) $\quad \mu \rightarrow \tilde{\mu}(\lambda)=\left\langle\mu(z), \frac{1}{\lambda-z}\right\rangle=\Sigma \mu_{n} \lambda^{2}$
iv) $\quad h(\lambda) \rightarrow \mu=\Sigma \frac{(-1)^{n}}{(n !)^{2}} h^{(-n)}(\infty) \delta^{(n)}$,
$E^{\prime} \rightarrow A$,
v) $h(\lambda) \rightarrow g(\xi)=\Sigma \frac{1}{(n !)^{2}} h^{(-n)}(\infty) \xi^{n}$,
$A \rightarrow E^{\prime}$,
vi) $g(\xi) \rightarrow h(\lambda)=\Sigma g^{(n)}(0) \lambda^{-n-1}$,
$A \rightarrow \operatorname{Exp}$,
$\operatorname{Exp} \rightarrow A$,

where $h^{(-n)}(\infty)=\left(\left(\frac{d}{d z}\right)^{n}\left(\frac{1}{z} h\left(\frac{1}{z}\right)\right)\right)_{z=0}$. Moreover, i) and ii), iii) and iv), and v) and vi) are inverses. That is,

vii) $\quad \mu=\Sigma \frac{(-1)^{n}}{n !} \hat{\mu}^{(n)}(0) \delta^{(n)}=\Sigma \frac{(-1)^{n}}{(n !)^{2}} \tilde{\mu}^{(-n)}(\infty) \delta^{(n)}$.

Also, we have representations:

viii) $\langle\mu, f\rangle=\Sigma \frac{\hat{\mu}^{(n)}(0) f^{(n)}(0)}{n !}$,

ix) $\langle\mu, f\rangle=\frac{1}{2 \pi i} \int_{|\lambda|=\boldsymbol{R}} \tilde{\mu}(\lambda) f(\lambda) d \lambda, \quad R$ sufficiently large,

x) $\hat{\mu}(\xi)=\frac{1}{2 \pi i} \int_{|\lambda|=R} \tilde{\mu}(\lambda) e^{\lambda \xi} d \lambda, \quad R$ sufficiently large.

Proof. As $e^{z \xi}=\Sigma \frac{z^{n} \xi^{n}}{n !}$ and the series converges uniformly on compact sets in $z$ we have $\hat{\mu}(\xi)=\Sigma \frac{\mu_{n} \xi^{n}}{n !}$ is entire. From $(2.4),|\hat{\mu}(\xi)| \leqq C\left\|e^{z \xi}\right\|_{N}$ $\leqq C e^{N|\xi|}$. For i), if $\hat{\mu}(\xi)=0$, then $\hat{\mu}^{(n)}(0)=\mu_{n}=\left\langle\mu, z^{n}\right\rangle=0$. But as the polynomials are dense in $E$, we have $\mu=0$.

Next, if $g \in \operatorname{Exp}$, then define $v=\Sigma \frac{(-1)^{n} g^{(n)}(0) \delta^{(n)}}{n !}$. If $g \in \operatorname{Exp}$, then from Cauchy's formula we have

$$
\left|g^{(n)}(0)\right| \leqq C n ! \frac{e^{S R}}{R^{n}} \leqq C S^{n} .
$$


Also, if $f \in E$, then

$$
\left|f^{(n)}(0)\right| \leqq C n ! \frac{\|f\|_{R}}{R^{n}} \text { for all } \quad R>0 .
$$

Consequently

$$
\begin{aligned}
|\langle v, f\rangle| & \leqq \Sigma \frac{\left|g^{(n)}(0)\right|\left|f^{(n)}(0)\right|}{n !} \\
& \leqq C\|f\|_{R} \Sigma(S / R)^{n} \leqq C_{1}\|f\|_{R}
\end{aligned}
$$

if $R$ is sufficiently large. Thus $v \in E^{\prime}$ and $\hat{v}=\hat{\mu}$ or $v=\mu$. This shows that ii) is the inverse of i) and maps Exp into $E^{\prime}$ completing i) and ii).

For iii), we have $\Sigma \mu_{n} \lambda^{-n-1}$ converges for $\lambda>S$ because $\mu_{n}=\hat{\mu}^{(n)}(0)$ satisfies (2.14). We get the same result if we note that (2.4) implies that $\mu$ can be extended to continuous linear functional on the space of functions analytic in $|z|<S$ and bounded for $|z| \leqq S$. If $\lambda>S$, then $\frac{1}{\lambda-z}$ is such a function. Clearly $\tilde{\mu}(\infty)=0$, i.e., $\tilde{\mu} \in A$. Again, if $\tilde{\mu}(\lambda)=0$, then $\mu_{n}=0$ and thus $\mu=0$. Next, suppose $h(\lambda) \in A$, i.e., $h(\lambda)=\sum_{n=0}^{\infty} a_{n} \lambda^{-n-1}$ converges for $|\lambda|>S$. Using 2.15 , we see that $\left|a_{n}\right| \leqq C S^{-n}$ and as in (2.16)

$$
v=\Sigma \frac{(-1)^{n} a_{n} \delta^{(n)}}{(n !)^{2}} \in E^{\prime}
$$

If $h(\lambda)=\tilde{\mu}(\lambda)$, then $\tilde{v}(\lambda)=\tilde{\mu}(\lambda)$, i.e. $v=\mu$. This completes iii) and iv). Also, v) and vi) are easy.

For vii), compute $\hat{\mu}$ and $\tilde{\mu}$ and then compare to the expressions in i) and iii). For viii), write $f$ in a power series. For ix), substitute expression 2.9 into vii) and check the uniform convergence of the series. For $\mathrm{x}$ ) set $f=e^{\lambda \xi}$ in ix).

\section{§ 3. Hyperdifferential Operators}

We wish to introduce a class of operators on $E$ or $E^{\prime}$ which includes differential operators and operators such as

$$
e^{\frac{d}{d z}}, e^{\left(\frac{d}{d z}+z\right)}, \sin \left(\frac{d}{d z}\right) .
$$

Also, we want the differential operators we will consider to be allowed to have variable coefficients which include at least the polynomials. 
First, in the standard way we define for $\mu \in E^{\prime}$ and $f \in E$

$$
\left\langle\frac{d \mu}{d z}, f(z)\right\rangle=-\left\langle\mu, \frac{d f}{d z}\right\rangle,\langle z \mu, f(z)\rangle=\langle\mu, z f(z)\rangle
$$

and, then note that, for example, $\delta^{(n)}=\left(\frac{d}{d z}\right)^{n} \delta$ as we defined $\delta^{(n)}$ in Section 2. Now we want a hyperdifferential operator on $E$ or $E^{\prime}$ to be given by an expression of the form

$$
A=\Sigma a_{m n} z^{m}\left(\frac{d}{d z}\right)^{n} .
$$

Definition. If $A$ is a bounded operator mapping $E$ into $E$ or $E^{\prime}$ into $E^{\prime}$, then $A$ is said to be hyperdifferential if

$$
A f(z)=\sum a_{m n} z^{m} f^{(n)}(z) \quad \text { or } \quad A \mu=\Sigma a_{m n} z^{m} \mu^{(n)}
$$

for some sequence $a_{m n} \in \mathbb{C}$.

Proposition. Every partial differential operator with polynomial coefficients is a hyperdifferential operator.

Proof. $\|z f\|_{R} \leqq R\|f\|_{R}$ and $\left\|\frac{d f}{d z}\right\|_{R} \leqq(S-R)^{-1}\|f\|_{S}$ if $S>R$, etc.

We now wish to define two symbols for $A$ and derive some of their properties.

Definition. If $A=\Sigma a_{m n} z^{m}\left(\frac{d}{d z}\right)^{n}$, then

$$
\begin{aligned}
& \hat{\sigma} A(z, \xi)=\Sigma a_{m n} z^{m} \xi^{n}=e^{-\xi z} A e^{\xi z} \\
& \tilde{\sigma} A(z, \lambda)=\Sigma(-1)^{n} n ! a_{m n} z^{m} \lambda^{-n-1}=\left.\Sigma a_{m n} z^{m}\left(\frac{d}{d w}\right)^{n} \frac{1}{\lambda-w}\right|_{w=0}
\end{aligned}
$$

where $\hat{\sigma}$ is called the symbol (or Fourier-Borel symbol) of $A$ and $\tilde{\sigma}$ is called the Cauchy Symbol of $A$.

Remark. If $A=P\left(\frac{d}{d z}\right)$ is a differential operator with constant coefficients, then $\hat{\sigma} A=P(\xi)$.

Theorem 3.1. The following are equivalent

i) $A=0$,

ii) $\hat{\sigma}(A)=0$,

iii) $\tilde{\sigma}(A)=0$,

if $A$ is a hyperdifferential operator on $E$. 
Proof. If $A=\Sigma a_{m n} z^{m}\left(\frac{d}{d z}\right)^{n}=0$, then $A(1)=\sum a_{m 0} z^{n}=0$ and consequently $a_{m 0}=0, m \geqq 0$. Similarly $A\left(z^{n}\right)=0$ implies $a_{m n}=0$ for all $m, n$. Thus i) $\Rightarrow$ ii).

Next, if $\hat{\sigma}(A)=0$, then $A e^{\xi z}=0=\Sigma \frac{A\left(z^{n}\right) \xi^{n}}{n !}$. Hence $A\left(z^{n}\right)=0$. However $\left\{z^{n}\right\}$ form a basis for $E$ and consequently $A=0$ so that ii) $\Rightarrow$ i). Obviously ii) and iii) are equivalent.

Theorem 3.2. The following conditions are equivalent:

i) $A$ is a hyperdifferential operator on $E$,

ii) $\hat{\sigma}(z, \xi)$ is entire in $z$ and $\xi$ and

$$
\sup _{|z| \leqq R}|\hat{\sigma} A(z, \xi)| \leqq C_{R} e^{S|\xi|} \quad \text { for all } R>0
$$

and some $S>R$.

iii) $\hat{\sigma}(z, \xi)=\sum a_{m n} z^{m} \xi^{n}$ and

$$
\left|a_{m n}\right| \leqq C_{R} R^{-m} S^{n}(n !)^{-1} \quad \text { for all } \quad R>0
$$

and some $S>R$.

iv) $\tilde{\sigma}(z, \lambda)$ is entire in $z$ and if $|z| \leqq R$, then there is an $S>R$ such that $\tilde{\sigma}(z, \lambda)$ is analytic in $\lambda$ for $|\lambda|>S$ and $\tilde{\sigma}(z, \infty)=0$.

Proof. If $A$ is a bounded operator on $E$, then $\left\|A e^{z \xi}\right\|_{R} \leqq C_{R}\left\|e^{z \xi}\right\|_{S}$ $\leqq C_{R} e^{S|\xi|}$ which shows i) $\Rightarrow$ ii).

From Cauchy's theorem we obtain

$$
\begin{aligned}
\left|a_{m n}\right| & \leqq C_{R} e^{S|\xi|} R^{-m}|\xi|^{-n} \\
& \leqq C_{R} S^{n} R^{-m}(n !)^{-1}
\end{aligned}
$$

which shows ii) $\Rightarrow$ iii). Clearly iii) $\Rightarrow$ iv).

Next we see that $\lambda^{-1} \tilde{\sigma}\left(z, \lambda^{-1}\right)$ is entire in $z$ and if $|z|<R$, then $\lambda^{-1} \tilde{\sigma}\left(z, \lambda^{-1}\right)$ is analytic for $|\lambda|<S^{-1}$. Again from Cauchy's theorem $\left|n ! a_{m n}\right| \leqq C_{R} R^{-m} S^{n}$. This shows iv) $\Rightarrow$ iii).

Also, if $O f=\sum a_{m n} z^{m} f^{(n)}(z)$, then

$$
\|O f\|_{R}=\sup _{|z|<R}|O f(z)| \leqq C_{R_{1}} \Sigma R_{1}^{-m} S_{1}^{n}(n !)^{-1} R^{m}(S-R)^{-n} n !\|f\|_{S} .
$$

Choosing $R_{1}>R$ and $S>R+S_{1}$ we have iii) $\Rightarrow$ i).

Corollary 3.1. If $A$ is a bounded operator on $E$, then $A$ is a hyperdifferential operator with $\sigma A(z, \xi)=e^{-\xi z} A e^{\xi z}$.

Proof. This is clear from Theorem 3.1 and Eq. (3.4).

At this point we want to write any hyperdifferential operator $A$ as an integral operator with kernel $a(z, w)$. 
We define in the usual way

$$
\begin{aligned}
& \langle\check{\mu}, f\rangle=\langle\mu(-z), f(z)\rangle=\langle\mu(z), f(-z)\rangle \\
& \langle\mu(z-w\rangle, f(z)\rangle=\langle\mu(z), f(z+w)\rangle \\
& \mu * f(z)=\langle\mu(z-w), f(w)\rangle=\langle\mu(w), f(z-w)\rangle
\end{aligned}
$$

and $\mu * v$ by

$$
\langle\mu * v, f\rangle=\langle\check{\mu}, v * f\rangle=\langle v, \check{\mu} * h\rangle
$$

where $f \in E$ and $\mu, v \in E^{\prime}$. The last definition makes sense because of:

Proposition. If $\mu \in E^{\prime}, f \in E$ then $\mu * f \in E$.

$$
\text { Proof. } f(w-z)=\Sigma \frac{f^{(n)}(w)(-z)^{n}}{n !} \text { or } \mu * f(w)=\Sigma \frac{(-1)^{n} \mu_{n} f^{(n)}(w)}{n !} \text {. }
$$

Now $\left\|f^{(n)}(w)\right\|_{R} \leqq C\|f\|_{2 R} R^{-n} n$ ! and $\left|\mu_{n}\right|<C S^{n}$ for some $S$ (see (2.14) and (2.15)) so that the series converges for all $w$. then

Corollary. If $k(z, w)=\Sigma f_{i}(z) \mu_{i}(w)$ with a finite sum and $f_{i} \in E, \mu_{i} \in E^{\prime}$,

$$
\begin{gathered}
A f(z)=\langle k(z, z-w), f(w)\rangle=\Sigma f_{i}(z) \mu_{i} * f(z) \\
A \mu(w)=\Sigma f_{i}(w) \mu_{i} * \mu(w)
\end{gathered}
$$

are hyperdifferential operators with

$$
\hat{\sigma} A(z, \xi)=\Sigma f_{i}(z) \hat{\mu}_{i}(-\xi) .
$$

Proof. Clear.

Remark. We can allow $k(z, w)$ to be an infinite sum (see [15]). we set

Definition. If $A=\sum a_{m n} z^{m}\left(\frac{d}{d z}\right)^{n}$ is a hyperdifferential operator, then

$$
a(z, w)=\sum a_{m n} z^{m} \delta^{(n)}(w)
$$

and call $a(z, w)$ the kernel of $A$. We then define

$$
\begin{aligned}
& k * f(z)=\langle k(z, w-z), f(w)\rangle=\Sigma a_{m n} z^{m} \delta^{(n)} * f(z)=\Sigma a_{m n} z^{m} f^{(n)}(z) \\
& k * \mu(z)=\Sigma a_{m n} z^{m} \delta^{(n)} * \mu(z)=\Sigma a_{m n} z^{m} \mu^{(n)}(z) .
\end{aligned}
$$

Proposition. If $f \in E, \mu \in E^{\prime}$ and $A$ is hyperdifferential operator, then

$$
\begin{aligned}
A f & =k * f, A \mu=k * \mu \\
\hat{\sigma}(A) & =\left\langle k(z, w), e^{-\xi w}\right\rangle, \tilde{\sigma}(A)=\left\langle k(z, w),(\lambda-w)^{-1}\right\rangle .
\end{aligned}
$$

Proposition. If $A$ is a hyperdifferential operator, then its kernel is unique.

Proof. This follows from Theorem 3.1. 
We note that we can find a hyperdifferential operator from its symbol via its kernel. There are other interesting formulas in $[15,16]$. We will give the following very useful representation.

Theorem 3.3. If $A$ is a hyperdifferential operator on $E^{\prime}$ and if $\mu \in E$ is given by a function with support on the real axis (i.e.,

$$
\langle\mu, f\rangle=\int \mu(x) f(x) d x, z=x+i y,
$$

where $\mu(x)$ is an integrable function with compact support), then

$$
A \mu(x)=(2 \pi)^{-1} \int e^{i x \xi} \sigma(x, i \xi) \hat{\mu}(-i \xi) d \xi .
$$

Proof.

$$
\begin{aligned}
\left\langle\left(\frac{d}{d z}\right)^{n} \mu, f\right\rangle & =(-1)^{n} \int \mu(x)\left(\frac{d}{d z}\right)^{n} f(z) d x=\int\left(\frac{d}{d x}\right)^{n} \mu(x) f(x) d x \\
& =\left\langle\left(\frac{d}{d x}\right)^{n} \mu(x), f(x)\right\rangle .
\end{aligned}
$$

The rest is now clear from the kernel formula.

Theorem 3.3 holds for distributions with compact support with the obvious modifications.

We now consider some algebraic properties of hyperdifferential operators.

Definition. If $A f=\Sigma a_{m n} z^{m}\left(\frac{d}{d z}\right)^{n} f$ is a hyperdifferential operator, then the transpose of $A$ is $A^{t} f=\Sigma(-1)^{n} a_{m n}\left(\frac{d}{d z}\right)^{n}\left(z^{m} f\right)$.

Theorem 3.4. If $A$ is a hyperdifferential operator on $E$ and for some $M$

$$
\sup _{|z|<R}|\hat{\sigma} A(z, \xi)| \leqq C_{R} \exp \left(M R^{a}|\xi|\right) \quad 0 \leqq a<1, \quad R \text { large, }
$$

then $A^{t}$ is a hyperdifferential operator in $E$ with

$$
\hat{\sigma} A^{t}(z, \xi)=e^{\partial_{z} \partial_{\xi}} \sigma A(z,-\xi), \quad \partial_{z}=\frac{\partial}{\partial z}, \quad \partial_{\xi}=\frac{\partial}{\partial \xi} .
$$

Proof. From Hormander [4, p. 10] we have Leibniz's rule

$$
\begin{aligned}
\left(\frac{d}{d z}\right)^{n}\left(z^{m} e^{\xi z}\right) & \left.=\Sigma\left(\frac{\partial^{\alpha}}{\partial z^{\alpha}} z^{m}\right)\left(\begin{array}{l}
n \\
\alpha
\end{array}\right)\left(\frac{d}{d z}\right)^{n-\alpha} e^{\xi z}\right) \\
& =\Sigma \frac{1}{\alpha !}\left(\frac{\partial^{2 \alpha}}{\partial z^{\alpha} \partial \xi^{\alpha}}\left(z^{m} \xi^{n}\right)\right) e^{\xi z} \\
& =\left(e^{\partial_{z} \partial \xi} z^{m} \xi^{n}\right) e^{\xi z} .
\end{aligned}
$$


If we sum over $n, m$ we get the result, provided $e^{\hat{\partial}_{z} \partial_{\xi}} \sigma A(z, \xi)$ is in fact the symbol of some bounded operator. To check this we compute:

$$
\begin{aligned}
& \sup _{|z|<R}\left|\partial_{z}^{\alpha} \partial_{\xi}^{\alpha} \sigma A(z, \xi)\right| \\
& \quad \leqq C(\alpha !)^{2}\left|\int_{\left|z_{1}\right|=R_{1}} \int_{\left|\xi-\xi_{1}\right|=S} \frac{\hat{\sigma} A\left(z_{1} \xi_{1}\right)}{\left(z_{1}-z\right)^{\alpha+1}\left(\xi_{1}-\xi\right)^{\alpha+1}} d \xi_{1} d z_{1}\right|, R_{1}>R \\
& \quad \leqq C(\alpha !)^{2} \exp \left(M R_{1}^{a}(|\xi|+S)\right)\left(R_{1}-R\right)^{-\alpha} S^{-\alpha} \\
& \leqq(\alpha !) \exp \left(M R_{1}^{a}|\xi|\right)(\alpha !) e^{\alpha}\left(M R_{1}^{a}\right)^{p} \alpha^{-\alpha}\left(R_{1}-R\right)^{-\alpha} \\
& \leqq(\alpha !) \exp \left(M R_{1}^{a}|\xi|\right)\left(M R_{1}^{a} /\left(R_{1}-R\right)\right)^{\alpha} .
\end{aligned}
$$

Thus the series for $\hat{\sigma} A^{t}(z, \xi)$ converges for $R_{1}=2 R, R^{1-a} \geqq 2^{a+1} M$ and thus

$$
\begin{aligned}
\sup _{|z|<R}\left|\hat{\sigma} A^{t}(z, \xi)\right| & \leqq C_{R} \exp \left(M R^{a}|\xi|\right) \text { for } R \text { sufficiently large } \\
& \leqq C_{R} e^{\varepsilon R|\xi|} \text { for any } \varepsilon \text { and all } R .
\end{aligned}
$$

Remark. Consider

$$
e^{\partial_{z} \partial_{\xi}} e^{z \xi}=\sum_{m \leqq n} \frac{1}{m !} \frac{1}{n !}\left(\frac{\partial^{2}}{\partial z \partial \xi}\right)^{m}(z \xi)^{n}=\sum_{m \leqq n} \frac{1}{n !} \frac{1}{m !}\left(\frac{n !}{(n-m) !}\right)^{2}(z \xi)^{n-m} .
$$

For $z$ and $\xi$, small and positive (or negative), this is a series of positive terms and can be summed any way we wish. Summing $n$ first we obtain $e^{\partial_{z} \partial_{\xi}} e^{z \xi}$. Summing $n=m$ we obtain $\infty$. Thus $e^{\partial_{z} \partial_{\xi}} e^{z \xi}$ is not an analytic function near $(0,0)$.

Definition. If $A$ is a bounded operator on $E$, then the operator $A^{*}$ operating on $E^{\prime}$ and defined by

$$
\left\langle A^{*} \mu, f\right\rangle=\langle\mu, A f\rangle
$$

is called the adjoint of $A$. Similarly if $A$ is defined on $E^{\prime}$, then $A^{*}$ is defined by

$$
\left\langle\mu, A^{*} f\right\rangle=\langle A \mu, f\rangle .
$$

Theorem 3.5. If $A$ is a hyperdifferential operator on $E$ and $\hat{\sigma}(A)$ satisfies 3.5, then $A^{*}$ is a hyperdifferential operator and $A^{*}=A^{t}$. A similar result holds if $A$ operates on $E^{\prime}$.

Proof. Clear.

Theorem 3.6. If $A$ and $B$ are hyperdifferential operators on $E$ (or $E^{\prime}$ ), then $A B$ ( $A$ composed with $B)$ is a hyperdifferential operator on $E$ (or $E^{\prime}$ ) 
with

$$
\begin{aligned}
\hat{\sigma}(A B)(z, \xi) & =\Sigma \frac{1}{\alpha !}\left(\partial_{\xi}\right)^{\alpha} \sigma A(z, \xi)\left(\partial_{z}\right)^{\alpha} \sigma B(z, \xi) \\
& =e^{\partial_{z} \partial_{\xi}}\left(A\left(z_{1}, \xi\right) B\left(z, \xi_{1}\right)\right)_{\substack{z_{1}=z \\
\xi_{1}=\xi}}
\end{aligned}
$$

Proof. We compute

$$
\begin{aligned}
\sup _{|z| \leqq R}|\hat{\sigma}(A \circ B)(z, \xi)| & \leqq \Sigma(p !) C_{R} S_{1}^{-p} e^{S\left(|\xi|+S_{1}\right)}\left(R_{1}-R\right)^{-p} e^{R_{1}|\xi|} \\
& \leqq C_{R} e^{\left(S+R_{1}\right)|\xi|} \Sigma\left(S_{1} /\left(R_{1}-R\right)\right)^{p}
\end{aligned}
$$

which is finite if we choose $R_{1}$ large enough. The rest is clear from Leibniz's rule.

Remark. For a more complete discussion of hyperdifferential operators see $[13,15,16]$.

\section{Applications to Quantum Mechanics}

We wish to consider the process of quantizing a classical function of the dynamical variables $q$ and $p$

$$
f(p, q)=\Sigma a_{m n} p^{m} q^{n} .
$$

It is well known that the formation of the corresponding quantum mechanical function presents difficulties because the coordinate and momentum operators $Q$ and $P$ do not commute. For convenience in what follows, we will take $P=\frac{d}{d z}, Q=z$ so that $[Q, P]=-1$. If in [17] one sets $\hbar=i$, one easily sees that the formulas there are the same as ours.

One particular way of quantizing functions is the Weyl prescription [17]

$$
\begin{aligned}
f(P, Q) & =\iint f(\sigma, \tau) e^{i(\sigma P+\tau Q)} d \sigma d \tau \\
f(\sigma, \tau) & =(2 \pi)^{-2} \iint f(p, q) e^{-i(\sigma p+\tau q)} d p d q .
\end{aligned}
$$

In the case $P=\frac{d}{d z}, Q=z$, we can formally compute the symbol of $f(P, Q)$ by

$$
\begin{aligned}
\sigma(f(P, Q))(z, \xi) & =e^{-z \xi} \iint \hat{f}(\sigma, \tau) e^{i\left(\sigma \frac{d}{d z}+\tau z\right)} e^{z \xi} d \sigma d \tau \\
& =e^{-z \xi} \iint \hat{f}(\sigma, \tau) e^{i \sigma \frac{d}{d z}} e^{i \tau z} e^{\sigma \tau / 2} e^{z \xi} d \sigma d \tau \\
& =\iint \hat{f}(\sigma, \tau) e^{-\sigma \tau / 2} e^{i \sigma \xi+i \tau z} d \sigma d \tau \\
& =e^{\frac{1}{2} \partial_{z} \partial_{\xi}} f(\xi, z)
\end{aligned}
$$


where we have used the Zassenhaus [17, Eq. (4.55)] formula in the second step.

If we write

$$
\sigma(z, \xi)=\sigma f(P, Q)(z, \xi)=\Sigma b_{m n} z^{m} \xi^{n}
$$

then

$$
f(P, Q)=\Sigma b_{m n} z^{m}\left(\frac{d}{d z}\right)^{n}
$$

as our symbolic calculus indicates. If we wish to compute the symbol of the transpose

$$
f^{t}(P, Q)=\Sigma(-1)^{n} b_{m n} \frac{d^{n}}{d z^{n}} z^{m}
$$

of $f(P, Q)$, then

$$
\begin{aligned}
\sigma\left(f^{t}(P, Q)\right)(z, \xi) & =e^{\partial_{z} \partial_{\xi}} \sigma(f(P, Q))(z,-\xi) \\
& =e^{\partial_{z} \partial_{\xi}+\frac{1}{2} \partial_{z} \partial_{\xi}} f(-\xi, z) \\
& =e^{\frac{3}{2} \partial_{z} \partial_{\xi}} f(-\xi, z)
\end{aligned}
$$

If we were to quantize the function

$$
g(p, q)=e^{\partial_{p} \partial_{q}} f(-p, q)
$$

then $g(P, Q)=f^{t}(P, Q)$.

Remark. We consider the Weyl quantization $f(P, Q)$ of $f(p, q)$ as having been arrived at by substituting $P$ for $p$ and $Q$ for $q$ in $f(p, q)$ with $f(p, q)$ written in a certain way, i.e. $f(p, q)=\sum a_{m n} p^{m_{1}} q^{n_{1}} \ldots p^{m_{2}} q^{n_{i}}$ with $m_{1}+\cdots+m_{i}=m n_{1}+\cdots+n_{i}=n$. If in $f(P, Q)$ we move all $P$ to the right of $Q$ using $[P, Q]=1$, we obtain an operator that is the same as substituting $P$ for $p$ and $Q$ for $q$ in $e^{\frac{1}{2} \partial_{p} \partial_{q}} f(p, q)$ with all $p$ written to the right of $q$ as indicated by Formula (4.4). On the other hand, if we wish to write $P$ to the left of $Q$, we substitute into $e^{\frac{3}{2} \partial_{p} \partial_{q}} f(p, q)$ with $p$ written to the right of $q$. This is the theorem of McCoy (see [17, p. 968]).

Remark. For additional methods of quantizing functions see [12].

Proposition. Suppose $C(P, Q), F(P, Q), G(P, Q)$ are the Weyl quantizations of $c(p, q) f(p, q)$ and $g(p, q)$ respectively and

$$
C(P, Q)=[F(P, Q), G(P, Q)],
$$

then

$$
c(p, q)=\left.2 \sinh \left(\frac{1}{2}\left(\partial_{p_{1}} \partial_{q_{2}}-\partial_{p_{2}} \partial_{q_{1}}\right)\right) f\left(p_{1} q_{1}\right) g\left(p_{2} q_{2}\right)\right|_{\substack{p_{1}=p_{2}=p \\ q_{1}=q_{2}=q}} .
$$

Remark. This is the theorem of Moyal (see [17, p. 968]). 
Proof. We have

$$
\begin{aligned}
C(z, \xi)= & \sum_{k} \frac{1}{k !}\left(\frac{\partial^{k} \sigma F}{\partial \xi^{k}} \frac{\partial^{k} \sigma G}{\partial z^{k}}-\frac{\partial^{k} \sigma G}{\partial \xi^{k}} \frac{\partial^{k} \sigma F}{\partial z^{k}}\right) \\
c(p, q)= & e^{-\frac{1}{2} \partial_{p} \partial_{q}} \sigma C(q, p) \\
= & e^{-\frac{1}{2} \partial_{p} \partial_{q}}\left(\sum_{p} \frac{1}{k !}\left(\frac{\partial}{\partial p}\right)^{k} e^{\frac{1}{2} \partial_{p} \partial_{q}} f(p, q)\left(\frac{\partial}{\partial q}\right)^{k} e^{\frac{1}{2} \partial_{p} \partial_{q}} g(p, q)-\cdots\right) \\
= & e^{-\frac{1}{2}\left(\partial_{p_{1}}+\partial_{p_{2}}\right)\left(\partial_{q_{1}}+\partial_{q_{2}}\right)} e^{\frac{1}{2}\left(\partial_{p_{1}} \partial_{q_{1}}+\partial_{p_{2}} \partial_{q_{2}}\right)} \\
& e^{\partial_{p_{1}} \partial_{q_{2}}} f\left(p_{1} q_{1}\right) g\left(p_{2}, q_{2}\right)-\cdots \\
= & e^{\frac{1}{2}\left(\partial p_{1} \partial_{q_{2}}-\partial_{p_{2}} \partial_{q_{1}}\right)} f\left(p_{1}, q_{1}\right) g\left(p_{2}, q_{2}\right)-\cdots \\
= & \left.2 \sinh \left(\frac{1}{2}\left(\partial_{p_{1}} \partial_{q_{2}}-\partial_{p_{2}} \partial_{q_{1}}\right)\right) f\left(p_{1} q_{1}\right) g\left(p_{2} q_{2}\right)\right|_{\substack{p_{1}=p_{2}=p \\
q_{1}=q_{2}=q}} .
\end{aligned}
$$

Next, if we consider quantizing the function $p^{\alpha} \phi(q)$ to the operator 0 , we see that $\sigma(0)=e^{\frac{1}{2} \partial_{\xi} \partial_{x}} \xi^{\alpha} \phi(x)$. On the other hand, if we define

$$
\begin{aligned}
& \{A, B\}^{\alpha}=\{A, B\}^{\alpha-1} B+B\{A, B\}^{\alpha-1} \quad \alpha>1 \\
& \{A, B\}^{1}=\{A, B\}=A B+B A,
\end{aligned}
$$

then one can easily compute by induction that

$$
\sigma\{P, \phi(Q)\}^{\alpha}=2^{\alpha} e^{\frac{1}{2} \partial_{\xi} \partial_{x}} \xi^{\alpha} \phi(x) .
$$

Consequently

$$
0=2^{-\alpha}\{P, \phi(Q)\}^{\alpha} .
$$

This is a theorem of Daughady and Nigam (see [17, p. 967]).

At this point we see how easily we can derive certain results in quantum mechanics. We now present some new results. It is clear that one can quantize any polynomial $f(p, q)$ using the Weyl prescription because

$$
e^{\frac{1}{2} \partial_{p} \partial_{q}} f(p, q)
$$

is a finite sum. On the other hand the Remark after Theorem 3.4 shows that one cannot quantize $e^{2 z \xi}$ as an operator with a symbol.

We see from Theorem 3.4 that if

$$
\sup _{|q|<R}|f(p, q)| \leqq C_{R} \exp \left(M R^{a}|p|\right)
$$

for some $M, a, 0 \leqq a<1$, then $f(p, q)$ can be quantized as an operator with a symbol. Also, in the proof of Theorem 5.1 in [13], there are given additional conditions under which a function $f(p, q)$ can be quantized with a symbol. These conditions essentially say that if one restricts the growth of $f(p, q)$ in the variable, say $p$, then one can allow $f(p, q)$ to grow fast in the variable $q$ or vice versa. 
At first these results seem to depend heavily on the choice of the space $E$. However, if one compares our theory to the theory in $[13,16]$ one sees that essentially for any choice of space in place of $E, e^{2 z \xi}$ cannot be quantized as an operator with a symbol which is analytic near zero.

We now consider the problem of normally ordering an operator. Suppose

$$
F(P, Q)=\Sigma a_{n} P^{n_{1}} Q^{n_{2}} \ldots P^{n_{k-1}} Q^{n_{k}}
$$

where the sum ranges over all $k$-tuples of non-negative integers for all non-negative values of $k$. We now want to find an expression

$$
F(P, Q)=\Sigma a_{i j} Q^{i} P^{j}
$$

for $F$ which is called the normally ordered form of $F(P, Q)$, see Section 10 and Footnote 62 in [17]. Now we have

$$
\sigma(F(P, Q))(x, \xi)=\sigma F(x, \xi)=\Sigma a_{i j} x^{i} \xi^{j} .
$$

Consequently, if we have an operator not in normal form and if we can compute $\sigma F=\sigma F(P, Q)=\Sigma b_{i j} x^{i} \xi^{j}$, then the normally ordered form of $F$ is given by

$$
F(P, Q)=\Sigma b_{i j} Q^{i} P^{j}
$$

Remark. Putting an operator such as (4.17) into normal form consists of moving all of the $P$ 's to the right of the $Q$ 's in expression (4.17) using the commutation rule $[P, Q]=1$.

As an example, consider the operator $P^{m} Q^{n}$ which we consider as the compose of $P^{m}$ and $Q^{n}$. Now $\sigma P^{m}=\xi^{m}$ and $\sigma Q^{n}=x^{n}$ and using the formulas for the symbol of a compose (3.15) we have

or

$$
\begin{aligned}
\sigma\left(P^{m} Q^{n}\right) & =\sum_{i} \frac{1}{i !}\left(\frac{\partial}{\partial \xi}\right)^{i} \xi^{m}\left(\frac{\partial}{\partial x}\right)^{j} x^{n} \\
& =\sum_{i=0}^{\min (m, n)} \frac{1}{i !} \frac{m !}{(m-i) !} \frac{n !}{(n-i) !} x^{n-i} \xi^{m-i}
\end{aligned}
$$

$$
P^{m} Q^{n}=\sum_{i=0}^{\min (m, n)} \frac{m ! n !}{i !(m-i) !(n-i) !} Q^{n-i} P^{m-i} .
$$

In fact, if we have the two operators $F=F(P, Q)$ and $G=G(P, Q)$ in normally ordered form, then $F G$ is not normally ordered. However our formula for the symbol of a compose gives

$$
\sigma(F G)=\Sigma \frac{1}{i !}\left(\frac{\partial}{\partial \xi}\right)^{i} \sigma F\left(\frac{\partial}{\partial x}\right)^{i} \sigma G
$$


which will put $F G$ in normally ordered form. Now, if we consider

$$
\begin{aligned}
\sigma F(x, \xi+ & \left.\frac{\partial}{\partial x}\right) \sigma G(x, \xi) \\
& =\Sigma \frac{1}{i !}\left(\frac{\partial}{\partial \xi}\right)^{i} \sigma F(x, \xi)\left(\frac{\partial}{\partial x}\right)^{i} \sigma G(x, \xi)
\end{aligned}
$$

where we have expanded $F(x, \xi)$ in a power series in the variable $\xi$ about the point $\xi$, we see that

$$
\sigma(F G)(x, \xi)=\sigma F\left(x, \xi+\frac{\partial}{\partial x}\right) \sigma G(x, \xi)
$$

which is equivalent to the Formula (10.6) in [17].

Next we observe that

$$
\begin{aligned}
\sigma e^{t(P+\phi(Q))} & =e^{-\xi x} e^{t\left(\frac{d}{d x}+\phi(x)\right)} e^{\xi x} \\
& =e^{t \xi} e^{t\left(\frac{d}{d x}+\phi(x)\right)}
\end{aligned}
$$

where again we have used the Zassenhaus formula [17, Eq. (4.55)]. If

$$
A=\sum_{n=1}^{\infty} \frac{\phi^{(n-1)}(x)}{n !}\left(\frac{\partial}{\partial \xi}\right)^{n},
$$

we see that

$$
\sigma e^{t(P+\phi(Q))}=e^{A} e^{t \xi}
$$

Also if $f(x)$ is a linear combination of $e^{t x}$, then clearly

$$
\sigma f(P+\phi(Q))=e^{A} f(\xi)
$$

and in particular

$$
\begin{aligned}
\sigma(P+Q)^{n} & =e^{x \frac{\partial}{\partial \xi}+\frac{1}{2} \frac{\partial^{2}}{\partial \xi^{2}}} \xi^{n} \\
& =\sum_{s=0}^{n-2 k} \sum_{k=0}^{[k / 2]} n ! \frac{x^{s} \xi^{n-2 k-s}}{s ! k !(n-2 k-s) !}
\end{aligned}
$$

which gives the Formulas (10.42b) and (10.43) in [17].

Thus we see that the process of computing a symbol of an operator is very useful but does not seem to be used in physics literature. 


\section{§ 5. Applications to Differential Equations}

In this section we want to solve differential equations of the form

$$
\begin{gathered}
\frac{\partial}{\partial t} P(x, y, t)=A P(x, y, t), \\
P(x, y, 0)=P_{0}(x, y)
\end{gathered}
$$

where $A$ is a differential operator with variable coefficients (i.e., depending on $x$ and $y$ ). This technique, called the symbolic calculus, has very general applications, and generalizes the Fourier transform techniques that may be used when $A$ has constant coefficients.

The solution to (5.1) can be written, at least formally, as $\exp (A t) P_{0}$. We call $\exp (A t)$ the resolvent operator of problem (5.1). In general, the resolvent is an integral operator and the symbolic calculus allows us to calculate its kernel (sometimes called the time dependent Green's function) independent of the initial data. We obtain our solution as an integral of the Fourier transform of the initial data and the kernel of the resolvent. We remark that once the resolvent kernel is obtained (i.e., the Green's function) much information can be read out of it, such as which class of initial data the problem can be solved for and for how long the solution exists as a classical function.

We are now going to work in two variables. That is, we will replace $z$ by $x$ and $y$, and $\xi$ by $\xi$ and $\eta$. We now write formulas (3.4) and (3.15) in this notation:

$$
\begin{gathered}
\sigma(H)(x, y, \xi, \eta)=\exp (-\xi x-\eta y) H \exp (\xi x+\eta y) \\
\sigma(H \circ K)(x, y, \xi, \eta)=\sum_{i, j=0}^{\infty} \frac{1}{i ! j !} \frac{\partial^{i+j} \sigma(H)}{\partial \xi^{i} \partial \eta^{j}} \frac{\partial^{i+j} \sigma(K)}{\partial x^{i} \partial y^{i}}
\end{gathered}
$$

where $H$ and $K$ are two hyperdifferential and operators and $H \circ K$ is $H$ composed with $K$. Also, if $A$ is a differential operator, then the symbol of the resolvent of $\exp (A t)$ is

$$
R(t, x, y, \xi, \eta)=\sigma(\exp (A t))(x, y, \xi, \eta)=e^{-\xi x-\eta y} e^{A t} e^{\xi x+\eta y}
$$

and satisfies the following differential equation:

$$
\begin{gathered}
\left(\frac{\partial}{\partial t}-A\right)[\exp (\xi x+\eta y) R(t, x, y, \xi, \eta)]=0 \\
R(0, x, y, \xi, \eta)=1 .
\end{gathered}
$$


Finally, if in problem (5.1) we can compute $\sigma(\exp (A t))$, then the solution of problem (5.1) is given by Eq. (3.8) in the form

$$
\begin{gathered}
P(x, y, t)=(2 \pi)^{-2} \int \exp (i(\xi x+\eta y)) R(t, x, y, i \xi, i \eta) \hat{P}_{0}(\xi, \eta) d \xi d \eta(5.6) \\
\hat{P}_{0}(\xi, \eta)=\int \exp (-i(\xi x+\eta y)) P_{0}(x, y) d x d y .
\end{gathered}
$$

We now proceed to solve problem (5.1) for a particular case. Consider the problem

$$
\begin{gathered}
\frac{\partial P}{\partial t}=\frac{\partial^{2} P}{\partial x \partial y}+a x \frac{\partial}{\partial x}+b y \frac{\partial}{\partial y}+c \quad(a, b, c \text { are constants }) \\
P(0, x, y)=P_{0}(x, y) .
\end{gathered}
$$

If we set

$$
A=\frac{\partial^{2}}{\partial x \partial y}+a x \frac{\partial}{\partial x}+b y \frac{\partial}{\partial y}+c
$$

we need to compute $\sigma(\exp (A t))$. We first note that if $[X, Y]=X Y-Y X$, then

$$
\begin{gathered}
{\left[\frac{\partial^{2}}{\partial x \partial y}, a x \frac{\partial}{\partial x}+b y \frac{\partial}{\partial y}+c\right]=(a+b) \frac{\partial^{2}}{\partial x \partial y}} \\
{\left[x \frac{\partial}{\partial x}, y \frac{\partial}{\partial y}\right]=\left[x \frac{\partial}{\partial x}, 1\right]=\left[y, \frac{\partial}{\partial y}\right]=0 .}
\end{gathered}
$$

From $[17]$ we find that if $[X, Y]=\alpha Y$, then

$$
\begin{aligned}
\exp (X+Y) & =\exp (X) \exp \left(\alpha^{-1}\left(1-e^{-\alpha}\right) Y\right) \\
& =\exp \left(\alpha^{-1}\left(e^{\alpha-1}-1\right) Y\right) \exp (X) .
\end{aligned}
$$

Consequently,

$\exp (A t)=\exp \left(\operatorname{tax} \frac{\partial}{\partial x}\right) \exp \left(t b y \frac{\partial}{\partial y}\right) \exp (c t) \exp \left(\left(\frac{e^{a+b}-1}{a+b}\right) t \frac{\partial^{2}}{\partial x \partial y}\right)$.

From (5.3) we obtain

$$
\sigma\left(\exp \left(\frac{e^{a+b}-1}{a+b} t \frac{\partial^{2}}{\partial x \partial y}\right)\right)=\exp \left(\frac{e^{a+b-} 1}{a+b} t \xi \eta\right) .
$$

Also, $\sigma\left(\right.$ at $\left.x \frac{\partial}{\partial x}\right)$ satisfies a first order differential equation given by (5.5), and this can be solved using the method of characteristics. However, 
we can use (5.8) to obtain the same result as follows:

$$
\begin{aligned}
& \sigma\left(\exp \left(\text { atx } \frac{\partial}{\partial x}\right)\right)(x, y, \xi, \eta) \\
& \quad=e^{-\xi x} \exp \left(\text { atx } \frac{\partial}{\partial x}\right) e^{\xi x} \\
& =e^{-\xi x} \exp \left(a t\left(x \frac{\partial}{\partial x}+\frac{\xi x}{1-\exp (-a t)}\right)\right) \\
& =e^{-\xi x} \exp \left(e^{a t} \xi x\right) \exp \left(a t x \frac{\partial}{\partial x}\right) \\
& =\exp \left(\left(e^{a t}-1\right) \xi x\right) .
\end{aligned}
$$

We now apply (5.4) to (5.10) obtaining

$$
\begin{aligned}
\sigma(A t) & =e^{c t} \sigma\left(\exp \left(\operatorname{tax} \frac{\partial}{\partial x}\right)\right) \sigma\left(\exp \left(\operatorname{tby} \frac{\partial}{\partial y}\right)\right) \sigma\left(\exp \left(\frac{e^{a+b}-1}{a+b} t \frac{\partial^{2}}{\partial x \partial y}\right)\right) \\
& =\exp \left\{c t+\left(e^{a t}-1\right) \xi x+\left(e^{b t}-1\right) \eta y+t \frac{e^{(a+b)}-1}{a+b} \xi \eta\right\} .
\end{aligned}
$$

The solution to problem (5.11) is now given by (5.6).

Remark. It is clear that $e^{A t}$ for our operator $A$ is not a hyperdifferential operator on $E$ as $\sigma e^{A t}$ does not have the correct growth properties. However $e^{A t}$ is a hyperdifferential operator on the spaces given in [13] and on those spaces has the symbol just calculated. Also the spaces used in [13] give the correct initial data for which problem (5.1) can be solved, see [3], Chapter 3.

We note that the above techniques solve the problems studied in $[2,6,8,10,11]$. An exposition of a general theory of the solution of problems like (5.7) appears in [13]. We remark that these techniques can be used to study equations whose coefficients also depend on time.

\section{§ 6. Summary}

At this point we have clearly demonstrated the usefulness of the theory of hyperdifferential operators and the symbolic calculus. The critical formulas that we use are the formula for the symbol (3.4), the integral representation (3.8), the symbol of the adjoint (3.10) and the symbol of the compose (3.15).

The choice of the space $E$ is not critical in this development. In fact, for discussing various problems it is essential to choose different spaces. 
In $[13,15,16]$ certain spaces of analytic or entire functions are chosen and particular applications are made to scales of Banach Spaces of functions. It is also possible to choose the spaces of type $W^{\Omega}$ in [3] or spaces of infinitely differentiable non-analytic functions of Gevrey type [1]. For each choice of space many technical changes must be made in the theory but the general aspects remain the same. Also, in [3] the Fourier dual corresponds to the Fourier-Borel transform of our dual spaces.

The solvability of the Cauchy problem for differential equations with constant coefficients is very well developed [3]. However, the theory presented here is specifically designed to handle the variable coefficient case. Compare the solution (5.6), (5.13) of Eqs. (5.1), (5.2) and the solution of the heat equation [3, p. 31, 37, 61, 111, 121, 164].

Another pleasant aspect of this theory is that the Formula (3.4) for the symbol of $A$ can be written $\hat{\sigma}(A)=e^{-B} A e^{B}$ where $B$ is the multiplication operator $\xi z$. This equation is in an advantageous form for applying Baker-Campbell-Housdorff [17] type formulas which we did extensively.

\section{References}

1. Du Chateau,P.: Thesis. Purdue University 1970.

2. Goldstein, M.E.: J. Math. Phys. 11, 667 (1970).

3. Gel'fand, I., Shilov, G.: Generalized functions. New York: Academic Press 1967.

4. Hormander, L.: Theory of partial differential equations.Berlin-Göttingen-Heidelberg: Springer 1963.

5. - Acta Math. to appear.

6. Kohn, J.J., Nirenberg, L.: Commun. Pure Appl. Math. 18, 269-305 (1965).

7. Kolsrud, M.: J. Math. Phys. 11, 829 (1970).

8. Lambropoulos, P.: J. Math. Phys. 8, 2167 (1967).

9. Mehta, C.L.: J. Math. Phys. 5, 677 (1964).

10. Miller, M. M.: J. Math. Phys. 10, 1406 (1969).

11. Nueringer, J. L.: J. Math. Phys. 10, 250 (1969).

12. Shewell, J.: Am. J. Phys. 27, 16 (1959).

13. Steinberg, S., Treves, F.: J. Diff. Eqns. 8, 333 (1970).

14. Treves, F.: Topological vector spaces, distributions and kernels. New York: Academic Press 1967.

15. - Ovcyannikov theorem and hyperdifferential operators. Rio de Janeiro: I.M.P.A. 1969.

16. - Bull. Soc. Math. France 97, 193 (1969).

17. Wilcox, R. M.: J. Math. Phys. 8, 962 (1967).

18. - J. Math. Phys. 11, 1235 (1970).

S. Steinberg

Division of Mathematical Sciences

Purdue University

Lafayette, Indiana, 47907, USA 